\title{
A Quasi-Projection Analysis of Galerkin Methods for Parabolic and Hyperbolic Equations
}

\author{
By Jim Douglas, Jr., Todd Dupont and Mary F. Wheeler*
}

\begin{abstract}
Superconvergence phenomena are demonstrated for Galerkin approximations of solutions of second order parabolic and hyperbolic problems in a single space variable. An asymptotic expansion of the Galerkin solution is used to derive these results and, in addition, to show optimal order error estimates in Sobolev spaces of negative index in multiple dimensions.
\end{abstract}

1. Introduction. We shall be concerned primarily with the analysis of superconvergence phenomena associated with the numerical solution of second order, linear parabolic and hyperbolic equations by Galerkin methods based on piecewise-polynomial spaces. Our principal tool will be an asymptotic expansion to high order of the Galerkin solution; this expansion will be obtained by using a sequence of elliptic projections and will be called a quasi-projection.

In Sections 4 and 5 we develop the quasi-projection for parabolic Galerkin procedures for problems in one or several space variables for both Neumann and Dirichlet boundary conditions and derive optimal order negative norm estimates for the error in the Galerkin solution. In Section 6 we apply the quasi-projection to derive superconvergence results in the case of a single space variable when the Galerkin space consists of piecewise-polynomial functions of degree $r$. It is well known [4], [6] , [7], [9], [10] that, if $h$ is the knot spacing parameter associated with the not necessarily uniform grid, the Galerkin solution for standard parabolic problems converges with an error that is at best globally of order $O\left(h^{r+1}\right)$, as measured in $L^{2}$ or $L^{\infty}$. Consider a knot at which the smoothness constraint of the Galerkin space reduces to continuity. We show that the Galerkin solution produces an $O\left(h^{2 r}\right)$-approximation at such a knot. Also, we show that a very simply evaluated weighted quadrature of the Galerkin solution gives an $O\left(h^{2 r}\right)$-approximation of the flux at the knot; the direct evaluation of the derivative of the Galerkin solution leads to an $O\left(h^{r}\right)$-approximation.

We summarize briefly in Section 7 results presented in detail elsewhere [3] showing that the superconvergence results above are preserved and that superconvergence occurs in the time increment when the Galerkin procedure is discretized in time by a collocation method.

In Section 8 we treat continuous-time Galerkin methods for hyperbolic problems and obtain analogous results.

Throughout this paper we rely heavily on some earlier results of two of the

Received March 21, 1975 ; revised July 11, 1977.

AMS (MOS) subject classifications (1970). Primary 65M15, 65N30.

* Research of the first two authors supported by NSF grant MCS74-12461. 
authors on two-point boundary problems [1] and on collocation methods for parabolic equations [2]. The quasi-projection is conceptually similar to the quasi-interpolant in [2] that played a central role in the derivation of superconvergence results in that treatment. The flux procedures evolve from earlier work by the other author [11] and her husband [8]. The applications of the quasi-projection have been limited to standard Galerkin methods in this paper; see [5], [12] for applications to $H^{1}$ and $H^{-1}$ Galerkin methods.

2. Some Notations and Preliminaries. Let $\Omega$ be a bounded domain in $\mathbf{R}^{\boldsymbol{n}}$ with a smooth boundary $\partial \Omega$; if $n=1$, let $\Omega=I$ be an interval. For nonnegative integers $s$ the real Sobolev space $H^{s}(\Omega)$ is the set of all real functions in $L^{2}(\Omega)$ whose distributional derivatives of order not greater than $s$ are also in $L^{2}(\Omega) ; H^{s}(\Omega)$ is normed by

$$
\|w\|_{s}^{2}=\sum_{|\alpha| \leqslant s}\left\|D^{\alpha} w\right\|^{2}
$$

where $\alpha=\left(\alpha_{1}, \alpha_{2}, \ldots, \alpha_{n}\right), \alpha_{i}$ a nonnegative integer, $|\alpha|=\alpha_{1}+\cdots+\alpha_{n}, D^{\alpha} w$ $=\partial^{|\alpha|} w / \partial x_{1}^{\alpha}{ }^{\alpha_{1}} \cdots \partial x_{n}^{\alpha_{n}}$, and

$$
\|z\|^{2}=\|z\|_{L^{2}(\Omega)}^{2}=\int_{\Omega} z^{2} d x
$$

Denote the inner products on the (real) spaces $L^{2}(\Omega)$ and $L^{2}(\partial \Omega)$ by

$$
(\varphi, \psi)=\int_{\Omega} \varphi \psi d x \quad \text { and }\langle\varphi, \psi\rangle=\int_{\partial \Omega} \varphi \psi d \sigma
$$

respectively. We shall also use the norm on the dual space $H^{-s}(\Omega)=\left(H^{s}(\Omega)\right)^{\prime}$; let

$$
\|\varphi\|_{-s}=\sup \left\{(\varphi, \psi) /\|\psi\|_{s}: \psi \in H^{s}(\Omega),\|\psi\|_{s} \neq 0\right\}
$$

Note that the duality is with $H^{s}(\Omega)$ and not with $H_{0}^{s}(\Omega)$. Finally, if $X$ is a normed space with norm $\|\cdot\|_{X}$ and if $\varphi:[0, T] \longrightarrow X$, we adopt the notations

$$
\|\varphi\|_{L^{2}(X)}^{2}=\|\varphi\|_{L^{2}(0, T ; X)}^{2}=\int_{0}^{T}\|\varphi(t)\|_{X}^{2} d t
$$

and

$$
\|\varphi\|_{L^{\infty}(X)}=\|\varphi\|_{L^{\infty}(0, T ; X)}=\underset{0 \leqslant t \leqslant T}{\operatorname{ess} \sup }\|\varphi(t)\|_{X} .
$$

The time interval $[0, T]$ will be denoted by $J$.

Let $a(x), b_{i}(x), 1 \leqslant i \leqslant n$, and $c(x)$ be $C^{\infty}(\bar{\Omega})$ functions, and assume that

$$
0<a_{0} \leqslant a(x) \leqslant a_{1}, \quad x \in \bar{\Omega} .
$$

Let $b(x)$ be the vector with components $b_{i}(x)$, and define the differential operator $L$ on $\Omega$ by

$$
L w=-\nabla \cdot(a(x) \nabla w)+b(x) \cdot \nabla w+c(x) w .
$$

The formal adjoint $L^{*}$ of $L$ is given by 


$$
L^{*} \varphi=-\nabla \cdot(a \nabla \varphi)-\nabla \cdot(b \varphi)+c \varphi .
$$

Let $B$ be the bilinear form associated with $L$ :

$$
B(\varphi, \psi)=(a \nabla \varphi, \nabla \psi)+(b \cdot \nabla \varphi+c \varphi, \psi)
$$

Assume that $B$ is coercive over $H^{1}(\Omega)$; i.e., assume that there exists a positive constant $b_{0}$ such that

$$
B(\varphi, \varphi) \geqslant b_{0}\|\varphi\|_{1}^{2}, \quad \varphi \in H^{1}(\Omega) .
$$

The assumption (2.3) is always obtainable by a trivial change of dependent variables in the nondegenerate parabolic case.

Galerkin methods require finite-dimensional subspaces of $H^{1}(\Omega)$ having good approximation properties. Let $0<h<1$ and associate a space $M_{h}$ with $h$ such that $M_{h}$ is a finite-dimensional subspace of $H^{1}(\Omega)$ and such that there exists a constant $C$, independent of $h$, for which

$$
\inf _{x \in M_{n}}\left\{\|\varphi-\chi\|+h\|\varphi-\chi\|_{1}\right\} \leqslant C h^{s}\left\|_{\varphi}\right\|_{s}
$$

for any $\varphi \in H^{s}(\Omega), 1 \leqslant s \leqslant r+1$, where $r$ is an integer greater than one. Set

$$
M_{h}^{0}=\left\{\chi \in M_{h}: \chi=0 \text { on } \partial \Omega\right\}
$$

and assume the approximation property that (2.4) holds for any $\varphi \in H^{s}(\Omega)$ such that $\varphi=0$ on $\partial \Omega$. Note that it is usually difficult to construct $M_{n}^{0}$ such that $(2.4)$ is valid for piecewise-polynomial spaces. We make this limiting assumption in this paper only because our applications are to problems with $\Omega=I$.

3. The Parabolic Problem and its Galerkin Method. We shall consider parabolic boundary problems with either Dirichlet or Neumann boundary conditions. Let $\rho \in$ $C^{\infty}(\bar{\Omega})$, where $0<\rho_{0} \leqslant \rho(x) \leqslant \rho_{1}$ on $\Omega$, and let $w$ satisfy

$$
\begin{gathered}
\rho \frac{\partial w}{\partial t}+L w=f, \quad(x, t) \in \Omega \times J, \\
w(0)=w^{*}, \quad x \in \Omega, \\
\alpha \frac{\partial w}{\partial v}+(1-\alpha) w=g, \quad(x, t) \in \partial \Omega \times J,
\end{gathered}
$$

where $\nu$ denotes the exterior normal to $\partial \Omega$, and $\alpha$ assumes the constant value zero or one for all $(x, t) \in \partial \Omega \times J$. If $\alpha=0$, assume $g=0$; i.e., we restrict ourselves to homogeneous Dirichlet data. Let

$$
M= \begin{cases}M_{h}^{0}, & \alpha=0 \\ M_{h}, & \alpha=1 .\end{cases}
$$

Then an integration by parts shows that

$$
(\rho \partial w / \partial t, v)+B(w, v)=(f, v)+\alpha(g, v\rangle, \quad v \in M
$$

A continuous-time Galerkin approximation to the solution of (3.1) can be defined 
by requiring that $w_{h}: J \rightarrow M$ satisfy

$$
\left(\rho \partial w_{h} / \partial t, v\right)+B\left(w_{h}, v\right)=(f, v)+\langle g, v\rangle, \quad v \in M, 0<t \leqslant T
$$

in addition, it is necessary to specify the initial condition $w_{h}(0)$. The initial condition will be given later (see (4.11)), since we have in mind a modification of the usual initial values in order to achieve superconvergence.

4. The Parabolic Quasi-Projection. The usual analysis [9] of the error in the Galerkin approximation for parabolic problems is founded upon comparing the function $w_{n}$ to the elliptic projection of the solution $w$ of (2.1). Let $\widetilde{w}_{h}: J \rightarrow M$ be given by

$$
B\left(\tilde{w}_{h}-w, v\right)=0, \quad v \in M, t \in J .
$$

If the initial condition for $w_{h}$ is chosen properly, it can be seen [6], [10] that $\widetilde{w}_{h}-$ $w_{h}$ is one power of $h$ smaller than either $\widetilde{w}_{h}-w$ or $w_{h}-w$ in both $L^{\infty}\left(L^{2}(\Omega)\right)$ and $L^{\infty}\left(H^{1}(\Omega)\right)$. This motivates the further use of elliptic projection to produce an approximation of $w_{h}$ to some higher order; it will be shown that an approximation of $w_{h}$ to order $O\left(h^{2 r}\right)$ can be obtained in the form of a finite expansion with the terms decreasing geometrically in size with ratio $O\left(h^{2}\right)$.

Let $z_{0}=\widetilde{w}_{h}-w, w_{0}=\widetilde{w}_{h}$, and $\theta_{0}=w_{0}-w_{h}$. Then it is easy to see from (3.3), (3.4), and (4.1) that

$$
\left(\rho \partial \theta_{0} / \partial t, v\right)+B\left(\theta_{0}, v\right)=\left(\rho \partial z_{0} / \partial t, v\right), \quad v \in M .
$$

Define maps $z_{j}: J \longrightarrow M$ recursively by

$$
B\left(z_{j}, v\right)=-\left(\rho \partial z_{j-1} / \partial t, v\right), \quad v \in M, t \in J, j=1,2, \ldots
$$

Set

$$
w_{j}=\tilde{w}_{h}+z_{1}+\cdots+z_{j}, \quad j \geqslant 1,
$$

and

$$
\theta_{j}=w_{j}-w_{h}, \quad j \geqslant 1 .
$$

A simple induction argument using (4.2) and (4.3) shows that

$$
\left(\rho \partial \theta_{j} / \partial t, v\right)+B\left(\theta_{j}, v\right)=\left(\rho \partial z_{j} / \partial t, v\right), \quad v \in M, t \in J
$$

The immediate objective is to show that the terms $z_{j}$ decrease for $J$ ranging to approximately $r / 2$. The argument will involve duality and estimates in Sobolev spaces of negative index in a serious way.

LEMma 4.1. Let $1 \leqslant q \leqslant r+1$ and assume that $\partial^{j+k} w / \partial t^{j+k} \in H^{q}(\Omega)$ for $t \in J$. Then, for $-1 \leqslant s$ and $\gamma=\min (s+2 j, r-1)$,

$$
\left\|\frac{\partial^{k} z_{j}}{\partial t^{k}}(t)\right\|_{-s} \leqslant C\left\|\frac{\partial^{j+k} w}{\partial t^{j+k}}(t)\right\|_{q} h^{q+\gamma}, \quad t \in J .
$$

Proof. Note first that the coefficients of $L$ are independent of $t$; hence 


$$
B\left(\frac{\partial^{k} z_{j}}{\partial t^{k}}, v\right)=-\left(\rho \frac{\partial^{k+1} z_{j-1}}{\partial t^{k+1}}, v\right), \quad v \in M
$$

It follows immediately from setting $v=\partial^{k} z_{j} / \partial t^{k}$ that

$$
\left\|\frac{\partial^{k} z_{j}}{\partial t^{k}}\right\|_{1} \leqslant C\left\|\frac{\partial^{k+1} z_{j-1}}{\partial t^{k+1}}\right\|_{-1}, \quad t \in J, j \geqslant 1 .
$$

Note that, if $r$ is even and (4.7) holds for $j<r / 2$, then it holds for $j=r / 2$. Consequently, it suffices to consider $s \leqslant r-2 j-1$.

Let $s \geqslant 0$ and assume that $\psi \in H^{s}(\Omega)$. Let $\varphi \in H^{s+2}(\Omega)$ be determined as the solution of the boundary problem for $L^{*} \varphi=\psi, x \in \Omega$, with

$$
a \frac{\partial \varphi}{\partial \nu}+\nu \cdot b \varphi=0, \quad x \in \partial \Omega, \text { if } \alpha=1,
$$

or

$$
\varphi=0, \quad x \in \partial \Omega \text {, if } \alpha=0 .
$$

In either case, $\|\varphi\|_{s+2} \leqslant C\|\psi\|_{s}$ by standard results on elliptic regularity.

First, consider $z_{0}=\widetilde{w}_{h}-w$. By the usual argument,

$$
\left(\frac{\partial^{m} z_{0}}{\partial t^{m}}, \psi\right)=\left(\frac{\partial^{m} z_{0}}{\partial t^{m}}, L^{*} \varphi\right)=B\left(\frac{\partial^{m} z_{0}}{\partial t^{m}}, \varphi\right)=B\left(\frac{\partial^{m} z_{0}}{\partial t^{m}}, \varphi-v\right), \quad v \in M .
$$

Hence, (2.4) implies that

$$
\left|\left(\frac{\partial^{m} z_{0}}{\partial t^{m}}, \psi\right)\right| \leqslant C\left\|\frac{\partial^{m} z_{0}}{\partial t^{m}}\right\|_{1} \inf _{v \in M}\|\varphi-v\|_{1} \leqslant C\left\|\frac{\partial^{m} z_{0}}{\partial t^{m}}\right\|_{1}\|\varphi\|_{s+2} h^{s+1}
$$

for $s+2 \leqslant r+1$ or $s \leqslant r-1$. Since the inequality

$$
\left\|\partial^{m} z_{0} / \partial t^{m}\right\|_{1} \leqslant C\left\|\partial^{m} w / \partial t^{m}\right\|_{q} h^{q-1}
$$

is trivial, (4.7) holds for $j=0$.

Next, it follows from (4.8) that

$$
\begin{aligned}
\left(\frac{\partial^{m} z_{j}}{\partial t^{m}}, \psi\right) & =B\left(\frac{\partial^{m} z_{j}}{\partial t^{m}}, \varphi-v\right)-\left(\rho \frac{\partial^{m+1} z_{j-1}}{\partial t^{m+1}}, v\right) \\
& =B\left(\frac{\partial^{m} z_{j}}{\partial t^{m}}, \varphi-v\right)+\left(\rho \frac{\partial^{m+1} z_{j-1}}{\partial t^{m+1}}, \varphi-v\right)-\left(\rho \frac{\partial^{m+1} z_{j-1}}{\partial t^{m+1}}, \varphi\right)
\end{aligned}
$$

Thus, (4.9) and (4.10) imply that

$$
\begin{aligned}
\left\|\frac{\partial^{m} z_{j}}{\partial t^{m}}\right\|_{-s} & \leqslant C\left\{\left\|\frac{\partial^{m} z_{j}}{\partial t^{m}}\right\|_{1} h^{s+1}+\left\|\frac{\partial^{m+1} z_{j-1}}{\partial t^{m+1}}\right\|_{-1} h^{s+1}+\left\|\frac{\partial^{m+1} z_{j-1}}{\partial t^{m+1}}\right\|_{-s-2}\right\} \\
& \leqslant C\left\{\left\|\frac{\partial^{m+1} z_{j-1}}{\partial t^{m+1}}\right\|_{-1} h^{s+1}+\left\|\frac{\partial^{m+1} z_{j-1}}{\partial t^{m+1}}\right\|_{-s-2}\right\}
\end{aligned}
$$


Since (4.7) holds for $j=0$, induction on $j$ implies that (4.7) holds, with the reduction on the range of $s$ in going from $j-1$ to $j$ being caused by the last term in the above inequality. Thus, the lemma has been proved.

Note that each $\partial^{k} z_{j} / \partial t^{k}$ is $O\left(h^{2 r}\right)$ in $H^{-s}(\Omega)$ for its maximum applicable value of $s$ if the solution $w$ is sufficiently smooth.

The estimates of Lemma 4.1 can be applied to (4.6) to bound the difference $\theta_{j}$ between $w_{j}$ and $w_{h}$ after a choice of the initial value of $w_{h}$ is made.

THEOREM 4.2. If $2 k \leqslant r-1$, let

$$
w_{h}(0)=w_{k}(0)=\widetilde{w}_{h}(0)+z_{1}(0)+\cdots+z_{k}(0),
$$

and let $1 \leqslant q \leqslant r+1$. Then,

$$
\left\|\theta_{k}\right\|_{L^{\infty}\left(L^{2}(\Omega)\right)} \leqslant C\left\|\frac{\partial^{k+1} w}{\partial t^{k+1}}\right\|_{L^{2}\left(H^{q}(\Omega)\right)} h^{q+\min (2 k+1, r-1)}
$$

and

$$
\left\|\theta_{k}\right\|_{L^{\infty}\left(H^{1}(\Omega)\right)} \leqslant\left\{\begin{array}{l}
C\left[\frac{\frac{\partial}{k+1} w_{\partial t^{k+1}} \|_{L^{2}\left(H^{q}(\Omega)\right)} h^{q+2 k}, \quad \text { if } 2 k \leqslant r-1,}{C\left[\left\|\frac{\partial^{k+1} w}{\partial t^{k+1}}\right\|_{L^{\infty}\left(H^{q}(\Omega)\right)}+\left\|\frac{\partial^{k+2} w}{\partial t^{k+2}}\right\|_{L^{1}\left(H^{q}(\Omega)\right)}\right.}\right] h^{q+2 k+1,} \\
\text { if } 2 k \leqslant r-2 .
\end{array}\right.
$$

Corollary 4.3. If $2 k \leqslant r-1,1 \leqslant q \leqslant r+1$, and $w_{h}(0)$ is defined by (4.11), then

$$
\left\|w-w_{h}\right\|_{L^{\infty}\left(H^{-s}(\Omega)\right)} \leqslant C h^{q+s} \sum_{j=0}^{k+1}\left\|\frac{\partial^{j} w}{\partial t^{j}}\right\|_{L^{2}\left(H^{q}(\Omega)\right)}
$$

for $0 \leqslant s \leqslant \min (2 k+1, r-1)$.

Proof of Theorem 4.2. Note that $\theta_{k}(0)=0$. To prove (4.12) choose $v=\theta_{k}$ in (4.6). Thus,

$$
\begin{aligned}
\frac{1}{2} \frac{d}{d t}\left(\rho \theta_{k}, \theta_{k}\right)+B\left(\theta_{k}, \theta_{k}\right) & =\left(\rho \frac{\partial z_{k}}{\partial t}, \theta_{k}\right) \\
& \leqslant C\left\|\frac{\partial z_{k}}{\partial t}\right\|_{-1}^{2}+\frac{1}{2} b_{0}\left\|\theta_{k}\right\|_{1}^{2}
\end{aligned}
$$

and (4.12) follows from (2.3) and Lemma 4.1. Next, choose $v=\partial \theta_{k} / \partial t$ in (4.6) and observe that

$$
B\left(\theta_{k}, \frac{\partial \theta_{k}}{\partial t}\right)=\frac{1}{2} \frac{d}{d t} B\left(\theta_{k}, \theta_{k}\right)-\frac{1}{2} \frac{d}{d t}\left(b \cdot \nabla \theta_{k}, \theta_{k}\right)+\left(b \cdot \nabla \theta_{k}, \frac{\partial \theta_{k}}{\partial t}\right) .
$$


Hence,

$$
\begin{aligned}
& \int_{0}^{t}\left(\rho \frac{\partial \theta_{k}}{\partial t}, \frac{\partial \theta_{k}}{\partial t}\right) d \tau+\frac{1}{2} B\left(\theta_{k}(t), \theta_{k}(t)\right) \\
& =\int_{0}^{t}\left(\rho \frac{\partial z_{k}}{\partial t}, \frac{\partial \theta_{k}}{\partial t}\right) d \tau+\frac{1}{2}\left(b \cdot \nabla \theta_{k}(t), \theta_{k}(t)\right)-\int_{0}^{t}\left(b \cdot \nabla \theta_{k}, \frac{\partial \theta_{k}}{\partial t}\right) d \tau
\end{aligned}
$$

If (2.3) is used along with the Gronwall inequality, it is easy to see that

$$
\begin{aligned}
\left\|\theta_{k}\right\|_{L^{\infty}\left(H^{1}(\Omega)\right)} & \leqslant C\left\{\left\|\theta_{k}\right\|_{L^{\infty}\left(L^{2}(\Omega)\right)}+\left\|\frac{\partial z_{k}}{\partial t}\right\|_{L^{2}\left(L^{2}(\Omega)\right)}\right\} \\
& \leqslant C\left\|\frac{\partial^{k+1} w}{\partial t^{k+1}}\right\|_{L^{2}\left(H^{q}(\Omega)\right)} h^{q+2 k},
\end{aligned}
$$

provided that $2 k \leqslant r-1$. This establishes the first inequality in (4.13). If $r$ is odd, the choices $2 k=r-1$ and $q=r+1$ produce an $O\left(h^{2 r}\right)$ estimate for $\theta_{k}$ in $H^{1}(\Omega)$; however, if $r$ is even, only an $O\left(h^{2 r-1}\right)$-estimate can be obtained from (4.15). The following argument regains the $O\left(h^{2 r}\right)$. Integrate the first right-hand side term in (4.14) by parts in time:

$$
\int_{0}^{t}\left(\rho \frac{\partial z_{k}}{\partial t}, \frac{\partial \theta_{k}}{\partial t}\right) d \tau=\left(\rho \frac{\partial z_{k}}{\partial t}(t), \theta_{k}(t)\right)-\int_{0}^{t}\left(\rho \frac{\partial^{2} z_{k}}{\partial t^{2}}, \theta_{k}\right) d \tau
$$

Then, if $\left\|\theta_{k}\left(t^{*}\right)\right\|_{1}$ maximizes the $H^{1}$-norm of $\theta_{k}(t)$,

$$
\left\|\theta_{k}\left(t^{*}\right)\right\|_{1}^{2} \leqslant C\left\{\left\|\frac{\partial z_{k}}{\partial t}\left(t^{*}\right)\right\|_{-1}^{2}+\left\|\theta_{k}\left(t^{*}\right)\right\|_{1} \int_{0}^{t^{*}}\left\|\frac{\partial^{2} z_{k}}{\partial t^{2}}\right\|\left\|_{-1} d \tau+\right\| \theta_{k}\left(t^{*}\right) \|^{2}\right\}
$$

and

$$
\begin{aligned}
& \left\|\theta_{k}\right\|_{L^{\infty}\left(H^{1}(\Omega)\right)} \leqslant C\left\{! \mid \frac{\partial z_{k}}{\partial t}\left\|_{L^{\infty}\left(H^{-1}(\Omega)\right)}+\right\| \frac{\partial^{2} z_{k}}{\partial t^{2}}\left\|_{L^{1}\left(H^{-1}(\Omega)\right)}+\right\| \theta_{k} \|_{L^{\infty}\left(L^{2}(\Omega)\right)}\right\} \\
& \quad \leqslant C\left\{\left\|\frac{\partial^{k+1} w}{\partial t^{k+1}}\right\|_{L^{\infty}\left(H^{q}(\Omega)\right)}+\left\|\frac{\partial^{k+2} w}{\partial t^{k+2}}\right\|_{L^{1}\left(H^{q}(\Omega)\right)}\right\} h^{q+2 k+1}
\end{aligned}
$$

for $2 k \leqslant r-2$. Thus, the theorem has been demonstrated.

For $t>0$ the functions $z_{j}$ are not, in general, computable; consequently, it is of interest to show that the initial condition (4.11) can be evaluated using no more than the data $f$ and $w^{*}$ and the differential operator. Let $k$ be chosen so that $k \leqslant$ $\bar{k}$, where

$$
2 \bar{k}= \begin{cases}r-1, & r \text { odd } \\ r-2, & r \text { even }\end{cases}
$$

In order to evaluate $z_{k}(0)$ using (4.3), it is necessary to evaluate $\partial z_{k-1}(0) / \partial t$ first, 
which in turn requires $\partial^{2} z_{k-2}(0) / \partial t^{2}, \ldots, \partial^{k} z_{0}(0) / \partial t^{k}$, by (4.8). Indeed, the evaluation of (4.11) necessitates performing the $1 / 2(k+1)(k+2)$ projections using $(4.1)$ or (4.8) to obtain $\partial^{m} z_{j}(0) / \partial t^{m}, 0 \leqslant j+m \leqslant k$. Now, $\partial^{m} w(0) / \partial t^{m}$ can be evaluated using $f, w^{*}$, and time-derivatives of the differential equation. Also, it follows from (4.1) that

$$
B\left(\frac{\partial^{m} \widetilde{w}_{h}}{\partial t^{m}}(0)-\frac{\partial^{m} w}{\partial t^{m}}(0), v\right)=0, \quad v \in M
$$

hence, the process can be started and then continued using (4.8) to find $z_{1}(0), \ldots$, $z_{k}(0)$. While the computational effort called for by (4.11) is obviously greater than that for the commonly used initial conditions, it is insignificant in terms of the overall problem. The code that takes time steps can be used to make the projections, so that the work for any single projection is about equivalent to that for a time step.

5. The Parabolic Quasi-Projection in a Single Space Variable. When $\Omega=I=$ $(0,1)$, Lemma 4.1 can be sharpened to give a bound on the value of $z_{j}$ at any knot $x_{i}$ at which the smoothness constraint reduces to simple continuity. Let $\Delta_{h}=\left\{x_{0}\right.$, $\left.x_{1}, \ldots, x_{N_{h}}\right\}, 0=x_{0}<x_{1}<\cdots<x_{N_{h}}=1$, with $\max \left(x_{i}-x_{i-1}\right)=h$, and assume that $M_{h}$ consists of piecewise-polynomial functions of degree $r$ having knots at $x_{i} \in \Delta_{h}$. At each $x_{i}, 1 \leqslant i \leqslant N_{h}-1$, the elements of $M_{h}$ will be assumed to be $C^{p_{i}}$. functions, $0 \leqslant p_{i}<r ; p_{0}$ and $p_{N_{h}}$ will be interpreted to be zero. Then (2.4) holds for both $M_{n}$ and $M_{n}^{0}$.

Let $\bar{x} \in[0,1]$, and assume that $\bar{x}=x_{i(n)}$ for each $h$ and that $p_{i(n)}=0$. In the discussion below, $\bar{x}$ will be treated as an interior point of $[0,1]$; the modification in the argument to allow $\bar{x}=0$ or $\bar{x}=1$ will be indicated at the end of the proof of Lemma 5.1 .

It is convenient to define some spaces that generalize $H^{s}(\Omega)$ in order to include a bound on the values of the functions at the point $\bar{x}$. For $s \geqslant 0$ let

$$
\widetilde{H}^{s}=\left\{u:\left.u\right|_{(0, \bar{x})} \in H^{s}((0, \bar{x})),\left.u\right|_{(\bar{x}, 1)} \in H^{s}((\bar{x}, 1))\right\} \times \mathbf{R},
$$

and set

$$
\|\|(u, \beta)\left\|_{s}^{2}=\right\| u\left\|_{H^{s}((0, \bar{x}))}^{2}+\right\| u \|_{H^{s}((\bar{x}, 1))}^{2}+\beta^{2} .
$$

For any elements $(u, \beta)$ and $(v, \gamma)$ of $\widetilde{H}^{0}$, define the inner product

$$
[(u, \beta),(v, \gamma)]=(u, v)+\beta \gamma,
$$

where $(u, v)$ denotes the usual $L^{2}(I)$-inner product. The space $\tilde{H}^{-s}, s \geqslant 0$, can be defined by duality with respect to the above inner product; however, since we shall need the dual norm only for functions in $H^{1}(I)$, we shall avoid some technicalities by restricting to such functions. Let $z \in H^{1}(I)$, and set

$$
\|\|_{z} \|_{-s}=\sup \left\{\frac{[(z, z(\bar{x})),(u, \beta)]}{\|\|(u, \beta)\|\|_{s}}:\|(u, \beta)\|_{s}>0\right\} .
$$


Note that the choice $u=0$ and $\beta=\operatorname{sgn} z(\bar{x})$ shows that

$$
|z(\bar{x})| \leqslant \|\left.\left.\right|_{z}||\right|_{-s}, \quad s \geqslant 0
$$

thus, the value at $\bar{x}$ of any function in $H^{1}(I)$ is majorized by any $\widetilde{H}^{s}$-norm, $s$ positive or negative. Hence, the following generalization of Lemma 4.1 provides excellent estimates for the functions $z_{j}(\bar{x})$ generated by (4.3).

Lemma 5.1. Let $1 \leqslant q \leqslant r+1$ and $0 \leqslant s \leqslant r-2 j-1$. If $\partial^{j+k} w(t) / \partial t^{j+k} \in$ $H^{q}(I)$ for $t \in J$, then

$$
\left\|\frac{\partial^{k} z_{j}}{\partial t^{k}}(t)\right\|\left\|_{-s} \leqslant C\right\| \frac{\partial^{j+k} w}{\partial t^{j+k}}(t) \|_{q} h^{q+s+2 j}, \quad t \in J .
$$

Proof. We shall use the $s=-1$ case covered by Lemma 4.1 . Let $s \geqslant 0$ and let $(\psi, \beta) \in \widetilde{H}^{s}$. Determine $\varphi \in C(I)$ as the solution of the two-point boundary problem given by

$$
\begin{aligned}
& L^{*} \varphi=\psi, \quad x \in I, \\
& \left(a \varphi^{\prime}+b \varphi\right)(x)=0, \quad x=0 \text { or } 1, \text { if } \alpha=1, \\
& \varphi(x)=0, \quad x=0 \text { or } 1, \text { if } \alpha=0, \\
& a \varphi^{\prime} \mid \frac{\bar{x}}{x}-0=-\beta .
\end{aligned}
$$

It is an exercise in ordinary differential equation theory to show that

$$
\|(\varphi, \varphi(\bar{x}))\|_{s+2} \leqslant C \mid\|(\psi, \beta)\|_{s},
$$

with the constant being independent of the position of $\bar{x}$. Integration by parts and the assumed jump condition on $\varphi$ lead to

$$
\begin{aligned}
& {\left[\left(\frac{\partial^{k} z_{j}}{\partial t^{k}}, \frac{\partial^{k} z_{j}}{\partial t^{k}}(\bar{x})\right),(\psi, \beta)\right]=B\left(\frac{\partial^{k} z_{j}}{\partial t^{k}}, \varphi\right)} \\
& =B\left(\frac{\partial^{k} z_{j}}{\partial t^{k}}, \varphi-v\right)+\left(\rho \frac{\partial^{k+1} z_{j-1}}{\partial t^{k+1}}, \varphi-v\right)-\left(\rho \frac{\partial^{k+1} z_{j-1}}{\partial t^{k+1}}, \varphi\right) .
\end{aligned}
$$

Since $\varphi \in C(I)$ is such that (with $M=M_{h}$ or $M_{h}^{0}$, as appropriate)

$$
\inf _{v \in M}\|\varphi-v\|_{1} \leqslant C\|\|(\varphi, \varphi(\bar{x}))\|\|_{s+2} h^{s+1}
$$

it follows that

$\left\|\frac{\partial^{k} z_{j}}{\partial t^{k}}\right\| \|_{-s} \leqslant C\left\{\left\|\frac{\partial^{k} z_{j}}{\partial t^{k}}\right\|_{1} h^{s+1}+\left.\left\|\frac{\partial^{k+1} z_{j-1}}{\partial t^{k+1}}\right\|\right|_{-1} h^{s+1}+\left\|\frac{\partial^{k+1} z_{j-1}}{\partial t^{k+1}}\right\| \|_{-s-2}\right\}$.

Note that (5.5) implies that \|\|$_{z}\left\|_{-s} \leqslant C\right\|_{z} \|_{-s}$. The first two terms on the righthand side have already been bounded in Lemma 4.1; if we can show that

$$
\left\|\frac{\partial^{k} z_{0}}{\partial t^{k}}\right\|\left\|_{-s} \leqslant C\right\| \frac{\partial^{k} w}{\partial t^{k}} \|_{q} h^{q+s}, \quad-1 \leqslant s \leqslant r-1,
$$


then the induction argument of Lemma 4.1 can be repeated to finish the proof. Since

$$
\left[\left(\frac{\partial^{k} z_{0}}{\partial t^{k}}, \frac{\partial^{k} z_{0}}{\partial t^{k}}(\bar{x})\right),(\psi, \beta)\right]=B\left(\frac{\partial^{k} z_{0}}{\partial t^{k}}, \varphi-v\right), \quad v \in M
$$

(5.9) follows from (5.8) and the known bound on $\left\|\partial^{k} z_{0} / \partial t^{k}\right\|_{1}$.

For Dirichlet boundary conditions it is pointless to consider $\bar{x}=0$ or 1 , but it is valuable to allow $\bar{x}$ to be an endpoint when Neumann boundary conditions are imposed. In this case the jump condition at $\bar{x}$ in the relations defining the function $\varphi$ modifies the boundary condition so that

$$
\left(a \varphi^{\prime}+b \varphi\right)(\bar{x})=\left\{\begin{aligned}
-\beta, & \text { if } \bar{x}=0, \\
\beta, & \text { if } \bar{x}=1,
\end{aligned}\right.
$$

and $a \varphi^{\prime}+b \varphi$ remains zero at the other end. Otherwise, the argument above is essentially unaffected, and Lemma 5.1 has been demonstrated.

Note that the choice $s=r-2 j-1$ in (5.6), combined with the inequality (5.5), shows that

$$
\left|z_{j}(\bar{x}, t)\right| \leqslant C\left\|\partial^{j} w(t) / \partial t^{j}\right\|_{q} h^{r+q-1}, \quad 1 \leqslant q \leqslant r+1, t \in J
$$

The remainder of this section is concerned with some estimates comparing Dirichlet and Neumann quasi-projections; these estimates will be applied in obtaining the flux estimates of the next section. Let $w$ be the solution of (3.1) with homogeneous Dirichlet data $(\alpha=0)$. Let $w_{h}$ be computed by (3.4) and (4.11), using $M=M_{h}^{0}$ and $\alpha=0$. Denote the corresponding quasi-projection by $w_{k}=\widetilde{w}_{h}+z_{1}+\cdots+$ $z_{k}$. Next, construct the Galerkin solution $u_{n}: J \rightarrow M_{n}$ by letting

$$
\left(\rho \frac{\partial u_{h}}{\partial t}, v\right)+B\left(u_{h}, v\right)=(f, v)+\left.a \frac{\partial w}{\partial x} v\right|_{0} ^{1}, \quad v \in M_{h}, 0<t \leqslant T
$$

where $u_{n}(0)$ is again determined by $(4.11)$, except that now the quasi-projection corresponds to the Neumann condition; i.e., let $u_{k}=\tilde{u}_{n}+y_{1}+\cdots+y_{k}$, where $\tilde{u}_{n}$ satisfies (4.1) with $M=M_{n}$ and $y_{j}$ satisfies (4.3). The function $u_{n}$ is being introduced for analytic purposes; it is not usually computable.

Let $z_{0}=\widetilde{w}_{h}-w, y_{0}=\tilde{u}_{h}-w$, and $\mu_{j}=y_{j}-z_{j}, j=0, \ldots, k$. Observe that

$$
B\left(\frac{\partial^{m} \mu_{0}}{\partial t^{m}}, v\right)=B\left(\frac{\partial^{m} \mu_{j}}{\partial t^{m}}, v\right)+\left(\rho \frac{\partial^{m+1} \mu_{j-1}}{\partial t^{m+1}}, v\right)=0, \quad v \in M_{h}^{0}
$$

First, choose

$$
v=\frac{\partial^{m} \mu_{0}}{\partial t^{m}}-\frac{\partial^{m} y_{0}}{\partial t^{m}}(0, t)(1-x)-\frac{\partial^{m} y_{0}}{\partial t^{m}}(1, t) x
$$

then, by earlier results on Galerkin methods for two-point boundary problems by two of the present authors [1], 


$$
\begin{aligned}
\left\|\frac{\partial^{m} \mu_{0}}{\partial t^{m}}\right\|_{1} & \leqslant C\left\{\left|\frac{\partial^{m} y_{0}}{\partial t^{m}}(0, t)\right|+\left|\frac{\partial^{m} y_{0}}{\partial t^{m}}(1, t)\right|\right\} \\
& \leqslant C\left\|\frac{\partial^{m} w}{\partial t^{m}}\right\|_{q} h^{r+q-1}, \quad t \in J .
\end{aligned}
$$

Next, choose

$$
v=\frac{\partial^{m} \mu_{j}}{\partial t^{m}}-\frac{\partial^{m} y_{j}}{\partial t^{m}}(0, t)(1-x)-\frac{\partial^{m} y_{j}}{\partial t^{m}}(1, t) x
$$

then, by Lemma 5.1 and (5.5),

$$
\begin{aligned}
\left\|\frac{\partial^{m} \mu_{j}}{\partial t^{m}}\right\|_{1} & \leqslant C\left\{\left|\frac{\partial^{m} y_{j}}{\partial t^{m}}(0, t)\right|+\left|\frac{\partial^{m} y_{j}}{\partial t^{m}}(1, t)\right|+\left\|\frac{\partial^{m+1} \mu_{j-1}}{\partial t^{m+1}}\right\|_{-1}\right\} \\
& \leqslant C\left\{\left\|\frac{\partial^{m+j} w}{\partial t^{m+j}}\right\|_{q} h^{r+q-1}+\left\|\frac{\partial^{m+1} \mu_{j-1}}{\partial t^{m+1}}\right\|_{1}\right\} .
\end{aligned}
$$

Induction applied to (5.13) and (5.14) shows that

$$
\left\|\frac{\partial^{m} \mu_{j}}{\partial t^{m}}\right\|_{1} \leqslant C\left\|\frac{\partial^{m+j} w}{\partial t^{m+j}}\right\|_{q} h^{r+q-1}, \quad t \in J, j=1, \ldots, k
$$

\section{Some Superconvergence Results for Parabolic Problems in a Single Space}

Variable. Let $M_{h}$ be the space described in the previous section, and assume that $\bar{x}$ $=x_{i(n)} \in \Delta_{h}$ is a knot for which $p_{i(n)}=0$. Let $w_{n}$ denote the Galerkin solution of the parabolic boundary problem (3.1) with either Dirichlet or Neumann boundary conditions, and assume that (4.11) defines the initial condition for the Galerkin solu. tion.

THEOREM 6.1. Let $1 \leqslant q \leqslant r+1$ and $2 k \leqslant r-1$. Then

$$
\left|\left(w-w_{h}\right)(\bar{x}, t)\right| \leqslant C \sum_{j=0}^{k}\left\|\frac{\partial^{j} w}{\partial t^{j}}\right\|_{L^{\infty}\left(H^{q}(I)\right)} h^{q+r-1}+\bar{e}
$$

where

$$
\bar{e}=\left\{\begin{array}{l}
C\left\|\frac{\partial^{k+1} w}{\partial t^{k+1}}\right\|_{L^{2}\left(H^{q}(I)\right)} h^{q+2 k}, \quad \text { if } 2 k \leqslant r-1, \\
C\left(\left\|\frac{\partial^{k+1} w}{\partial t^{k+1}}\right\|_{L^{\infty}\left(H^{q}(I)\right)}+\left\|\frac{\partial^{k+2} w}{\partial t^{k+2}}\right\|_{L^{1}\left(H^{q}(I)\right)}\right) h^{q+2 k+1},
\end{array}\right.
$$

$$
\text { if } 2 k \leqslant r-2 \text {. }
$$

Proof. Write $w-w_{h}$ in the form

$$
\left(w-w_{h}\right)(\bar{x}, t)=\left(w-\widetilde{w}_{h}\right)(\bar{x}, t)+\left(w_{k}-w_{h}\right)(\bar{x}, t)-\sum_{j=1}^{k} z_{j}(\bar{x}, t) .
$$


It is known [1] (and follows from (5.10), as well) that $\left(w-\widetilde{w}_{h}\right)(\bar{x}, t)$, which is just the error in the Galerkin solution of the two-point boundary problem associated with the operator $L$ and whichever boundary conditions are assigned, is bounded at a knot at which $p_{i(n)}=0$ by

$$
\left|\left(w-\widetilde{w}_{h}\right)(\bar{x}, t)\right| \leqslant C\|w(t)\|_{q} h^{q+r-1}, \quad t \in J .
$$

The second term is bounded by $\bar{e}$ as a consequence of the $H^{1}(\Omega)$-estimate of Theorem 4.2, and (5.10) completes the demonstration.

A slightly less precise statement of the result when $k$ takes on its maximum useful value is given by

$$
\left|\left(w-w_{h}\right)(\bar{x}, t)\right| \leqslant C \sum_{j=0}^{[r / 2\}+1}\left\|\frac{\partial^{j} w}{\partial t^{j}}(t)\right\|_{L^{\infty}\left(H^{q}(I)\right)} h^{q+r-1}, \quad t \in J .
$$

The superconvergent approximation of the knot value results from evaluating the Galerkin solution at the knot. Other superconvergent approximations of quantities associated with the solution of the differential equation can be formed by simple manipulations of the Galerkin solution. Consider (3.1) with homogeneous Dirichlet data, and let us produce an $O\left(h^{2 r}\right)$-approximation of the flux across the boundary at $x=1$. If the Galerkin solution is differentiated and evaluated at $x=1$, only an $O\left(h^{r}\right)$-approximation will be obtained.

To motivate the selection of a relation to find the flux, consider the function $u_{n}: J \rightarrow M_{h}$ defined by (5.11) and use $v(x)=x$ as the test function in (5.11). Then,

$$
(a \partial w / \partial x)(1, t)=\left(\rho \partial u_{h} / \partial t, x\right)+B\left(u_{h}, x\right)-(f, x) .
$$

The function $u_{n}$ is not computable, but we have seen that $u_{n}$ and $w_{n}$, the Galerkin solution with the Dirichlet data, are very nearly the same. Thus, we let

$$
\Gamma_{1}(t)=\left(\rho \partial w_{h} / \partial t, x\right)+B\left(w_{h}, x\right)-(f, x), \quad t \in J .
$$

Note that $\Gamma_{1}$ need be evaluated only at times at which the flux is desired. Moreover, since any function in $M_{h}^{0}$ can be added to $v(x)=x$ without changing the right-hand side, we can for the purpose of evaluating $\Gamma_{1}$ take

$$
\Gamma_{1}(t)=\left(\rho \partial w_{h} / \partial t, \gamma\right)+B\left(w_{h}, \gamma\right)-(f, \gamma), \quad t \in J
$$

where

$$
\gamma(x)=\left\{\begin{array}{l}
\frac{\left(x-x_{N_{h}-1}\right)^{m}}{\left(1-x_{N_{h^{-1}}}\right)^{m}}, \quad x_{N_{h^{-1}}} \leqslant x \leqslant 1, \\
0, \text { otherwise, }
\end{array}\right.
$$

where $m=1+p_{N_{h}-1}$. Observe that (6.9) requires the evaluation of quadratures over a single subinterval and, consequently, is a completely trivial calculation.

To analyze the error in the approximation of the flux, first put $\xi=w_{n}-u_{n}$. 
Then, (6.6) and (6.7) imply that

$$
\Gamma_{1}(t)-(a \partial w / \partial x)(1, t)=(\rho \partial \xi / \partial t, x)+B(\xi, x)
$$

Now,

$$
B(\xi, x)=\left(\xi,-(a+x b)^{\prime}+c x\right)+\left.(a+x b) \xi\right|_{0} ^{1}
$$

and it follows that

$$
\left|\Gamma_{1}(t)-\left(a \frac{\partial w}{\partial x}\right)(1, t)\right| \leqslant C\left\{|| \frac{\partial \xi}{\partial t}\|+\| \xi \|+|\xi(0, t)|+|\xi(1, t)|\right\} .
$$

If we adopt the notations used toward the end of Section 5 and in addition set $\psi_{k}=$ $u_{k}-u_{n}$ and $\theta_{k}=w_{k}-w_{h}$, then

$$
\xi=\psi_{k}-\theta_{k}-\mu_{0}-\sum_{j=1}^{k} \mu_{j}
$$

Theorem 4.2, (5.13), and (5.15) show that

(6.12) $\|\xi\| \leqslant C \sum_{j=0}^{k}\left\|\frac{\partial^{j} w}{\partial t^{j}}(t)\right\| h_{q} h^{q+r-1}+C\left\|\frac{\partial^{k+1} w}{\partial t^{k+1}}\right\|_{L^{2}\left(H^{q}(I)\right)} h^{q+\min (2 k+1, r-1)}$.

The estimation of $\partial \xi / \partial t$ requires that $\partial \psi_{k} / \partial t$ and $\partial \theta_{k} / \partial t$ be bounded. First, it follows from (4.6) and the fact that $\psi_{k}(0)=\theta_{k}(0)=0$ that

$$
\begin{aligned}
\left\|\frac{\partial \theta_{k}}{\partial t}(0)\right\|+\left\|\frac{\partial \psi_{k}}{\partial t}(0)\right\| & \leqslant C\left(\left\|\frac{\partial z_{k}}{\partial t}(0)\right\|+\left\|\frac{\partial y_{k}}{\partial t}(0)\right\|\right) \\
& \leqslant C\left\|\frac{\partial^{k+1} w}{\partial t^{k+1}}(0)\right\|_{h^{q+\beta}}
\end{aligned}
$$

for $1 \leqslant q \leqslant r+1$ and $\beta=\min (2 k, r-1)$. Note that (4.6) can be differentiated with respect to time and then $v$ chosen to be (for the case of the $\partial \theta_{k} / \partial t$ estimate) $\partial \theta_{k} / \partial t$. Then, it is easy to see that

$$
\begin{aligned}
\left\|\frac{\partial \theta_{k}}{\partial t}\right\|_{L^{\infty}\left(L^{2}(I)\right)} & \leqslant C\left\{\left\|\frac{\partial \theta_{k}}{\partial t}(0)\right\|+\left\|\frac{\partial^{2} z_{k}}{\partial t^{2}}\right\|_{L^{2}\left(H^{-1}(I)\right)}\right\} \\
& \leqslant C\left\{\left\|\frac{\partial^{k+1} w}{\partial t^{k+1}}(0)\right\|_{q}+\left\|\frac{\partial^{k+2} w}{\partial t^{k+2}}\right\|_{L^{2}\left(H^{q}(I)\right)}\right\} h^{q+\beta} .
\end{aligned}
$$

Hence,

$$
\left\|\frac{\partial \xi}{\partial t}\right\| \leqslant C \sum_{j=1}^{k+1}\left\|\frac{\partial^{j} w}{\partial t^{j}}(t)\right\|_{q} h^{q+r-1}
$$

$$
+C\left\{\left\|\frac{\partial^{k+1} w}{\partial t^{k+1}}(0)\right\|_{q}+\left\|\frac{\partial^{k+2} w}{\partial t^{k+2}}\right\|_{L^{2}\left(H^{q}(I)\right)}\right\} h^{q+\beta}
$$


Since $\xi(0, t)=-u_{n}(0, t)$ and $\xi(1, t)=-u_{n}(1, t)$, Theorem 6.1 implies that

$$
\begin{aligned}
& |\xi(0, t)|+|\xi(1, t)| \\
& \leqslant C \sum_{j=0}^{k}\left\|\frac{\partial^{j} w}{\partial t^{j}}(t)\right\|_{q} h^{q+r-1}+C\left\|\frac{\partial^{k+1} w}{\partial t^{k+1}}\right\|_{L^{\infty}\left(H^{q}(I)\right)} h^{q+\beta} .
\end{aligned}
$$

The inequalities (6.12)-(6.14) combine to give the following theorem.

THEOREM 6.2. Let $w_{h}: J \rightarrow M_{h}^{0}$ be the solution of (3.4) and (4.11), and let $\Gamma_{1}(t)$ be defined by either of the equivalent relations (6.7) and (6.8). If $1 \leqslant q \leqslant r$ +1 and $\beta=\min (2 k, r-1)$, then

$$
\left|\Gamma_{1}(t)-\left(a \frac{\partial w}{\partial x}\right)(1, t)\right|
$$

$$
\begin{aligned}
\leqslant C\left\{\sum_{j=0}^{k} \|\right. & \frac{\partial^{j} w}{\partial t^{j}}(t) \|_{q} h^{q+r-1} \\
& \left.+\left(\left\|\frac{\partial^{k+1} w}{\partial t^{k+1}}\right\|_{L^{\infty}\left(H^{q}(I)\right)}+\left\|\frac{\partial^{k+2} w}{\partial t^{k+2}}\right\|_{L^{2}\left(H^{q}(I)\right)}\right) h^{q+\beta}\right\} .
\end{aligned}
$$

In particular, if $r$ is odd and $2 k=r-1$ or if $r$ is even and $2 k=r$,

$$
\left|\Gamma_{1}(t)-\left(a \frac{\partial w}{\partial x}\right)(1, t)\right| \leqslant C \sum_{j=0}^{k+2}\left\|\frac{\partial^{j} w}{\partial t^{j}}\right\|_{L^{2}\left(H_{(I))}\right.} h^{q+r-1}, \quad 1 \leqslant q \leqslant r+1 .
$$

It is also possible to produce an $O\left(h^{2 r}\right)$-approximation of the flux at an interior point of $I$. Let $\bar{x}=x_{i(h)} \in \Delta_{h}$ and assume, as usual, that $p_{i(h)}=0$. Let

$$
\Gamma_{\bar{x}}(t)=\bar{x}^{-1}\left\{\left(\rho \partial w_{h} / \partial t, x\right)_{\bar{x}}+B_{\bar{x}}\left(w_{h}, x\right)-(f, x)_{\bar{x}}\right\},
$$

where the subscript $\bar{x}$ indicates that the integrals are to be taken over the interval $(0, \bar{x})$. If

$$
\delta(x)=\left\{\begin{array}{l}
\left(x_{i(n)}-x_{i(n)-1}\right)^{-r}\left(x-x_{i(n)-1}\right)^{r}, \quad x_{i(h)-1} \leqslant x \leqslant x_{i(n)}, \\
0, \text { otherwise, }
\end{array}\right.
$$

then $\Gamma_{\bar{x}}(t)$ can be evaluated using quadratures over a single subinterval by

$$
\Gamma_{\bar{x}}(t)=\left(\rho \partial w_{h} / \partial t, \delta\right)+B\left(w_{h}, \delta\right)-(f, \delta) .
$$

Clearly, by interchanging $x$ for $1-x$, an approximation to $(a \partial w / \partial x)(x, t)$ can be obtained using the subinterval to the right of $x_{i(n)}$ instead of the one to the left. Inequalities analogous to (6.15) and (6.16) can be proved for $\left|\Gamma_{\bar{x}}(t)-(a \partial w / \partial x)(\bar{x}, t)\right|$. The proof is quite similar to that given above, with the function $u_{n}$ being replaced by $s_{h}: J \rightarrow M_{h, \bar{x}}=\left\{\left.v\right|_{[0, \bar{x}]}: v \in M_{h}\right\}$ where $s_{h}$ satisfies

$$
\left(\rho \frac{\partial s_{h}}{\partial t}, v\right)_{\bar{x}}+B_{\bar{x}}\left(s_{h}, v\right)=(f, v)_{\bar{x}}+\left.\left(a \frac{\partial w}{\partial x} v\right)\right|_{0} ^{\bar{x}}, \quad v \in M_{h, \bar{x}}
$$


with $s_{n}(0)$ being determined by the analogue of $(4.11)$ for $(0, \bar{x})$. A little care shows that the corresponding constants $C$ are independent of $\bar{x}$.

7. Discretization in Time for the Parabolic Problem. A detailed description of the discretization in time of the Galerkin method (3.4)-(4.11) using collocation at Gaussian points in time for a solution space that is the tensor product of $M_{n}$ or $M_{n}^{0}$ in space with $C^{0}$ piecewise-polynomial functions in time is presented in the authors' Mathematics Research Center technical report [3]. The superconvergence results of Section 6 are generalized in a way that covers the knot value results, with superconvergence occurring in the time increment as well as $h$, and that produces flux estimates related to $(6.15)$. The results pertain to Crank-Nicolson differencing as a special case. The development strongly resembles that given earlier by two of the authors for collocation-collocation methods for parabolic equations [2]

\section{The Quasi-Projection and Superconvergence for Galerkin Methods for the} Wave Equation. We shall restrict our attention to the single space variable case of $\Omega=I=(0,1)$ and to the space $M_{n}$ of piecewise-polynomial functions of degree $r$ described in Section 5. Throughout this section let $\bar{x}=x_{i(n)} \in \Delta_{h}$ and assume that $p_{i(h)}=0$. Let $w$ satisfy the wave equation subject to homogeneous Dirichlet boundary values:

$$
\begin{aligned}
& \rho \frac{\partial^{2} w}{\partial t^{2}}+L w=f, \quad(x, t) \in I \times J, \\
& w(0, t)=w(1, t)=0, \quad t \in J, \\
& w(x, 0)=w_{0}^{*}(x), \quad \frac{\partial w}{\partial t}(x, 0)=w_{1}^{*}(x), \quad x \in I .
\end{aligned}
$$

A continuous-time Galerkin method can be defined for (8.1) by seeking $w_{n}: J \longrightarrow M$ $=M_{n}^{0}$ such that

$$
\left(\rho \partial^{2} w_{h} / \partial t^{2}, v\right)+B\left(w_{h}, v\right)=(f, v), \quad v \in M, t \in J
$$

where $w_{h}(0)$ and $\partial w_{h} / \partial t(0)$ will be specified later (see (8.6)).

A quasi-projection for the hyperbolic problem can be constructed in a manner analogous to that used in Section 4. Let $\widetilde{w}_{h}: J \rightarrow M$ be the elliptic projection of the solution $w$ :

$$
B\left(\tilde{w}_{h}-w, v\right)=0, \quad v \in M, t \in J
$$

Set $z_{0}=\widetilde{w}_{n}-w$ and recursively define $z_{j}: J \longrightarrow M$ by the relation

$$
B\left(z_{j}, v\right)=-\left(\rho \partial^{2} z_{j-1} / \partial t^{2}, v\right), \quad v \in M, t \in J
$$

Let $w_{k}=\tilde{w}_{h}+z_{1}+\cdots+z_{k}$ and $\theta_{k}=w_{k}-w_{h}$. It is easy to show inductively that

$$
\left(\rho \frac{\partial^{2} \theta_{k}}{\partial t^{2}}, v\right)+B\left(\theta_{k}, v\right)=\left(\rho \frac{\partial^{2} z_{k}}{\partial t^{2}}, v\right), \quad v \in M, t \in J
$$


We shall utilize the quasi-projection $w_{k}$ to set up initial conditions for (8.2); let

$$
w_{h}(0)=w_{k}(0), \quad \frac{\partial w_{h}}{\partial t}(0)=\frac{\partial w_{k}}{\partial t}(0), \quad 2 k \leqslant r-1 .
$$

The function $w_{h}(0)$ can be evaluated using $w_{0}^{*}$ and the differential equation, and $\partial w_{h} / \partial t(0)$ using $w_{1}^{*}$ and the differential equation.

An argument paralleling that leading to Lemmas 4.1 and 5.1 leads to the following bounds for $t \in J$ and $1 \leqslant q \leqslant r+1$ :

$$
\left\|\frac{\partial^{m} z_{j}}{\partial t^{m}}\right\|_{-s} \leqslant C\left\|\frac{\partial^{2 j+m} w}{\partial t^{2 j+m}}\right\|_{q} h^{q+s+2 j}, \quad 0 \leqslant j<\frac{r}{2},-1 \leqslant s \leqslant r-2 j-1,
$$

$$
\left|\frac{\partial^{m} z_{j}}{\partial t^{m}}(\bar{x}, t)\right| \leqslant C\left\|\frac{\partial^{2 j+m} w}{\partial t^{2 j+m}}\right\|_{q} h^{q+r-1}, \quad 0 \leqslant j \leqslant \frac{r}{2} .
$$

The total energy is the most natural measure to associate with solutions of wave equations. Hence, use $v=\partial \theta_{k} / \partial t$ in (8.5) and note that (8.6) implies that $\theta_{k}(0)=$ $\partial \theta_{k} / \partial t(0)=0 ;$ thus, if

$$
|u|_{m}^{2}=\sum_{\alpha+\beta \leqslant m}\left\|\frac{\partial^{\alpha+\beta} u}{\partial x^{\alpha} \partial t^{\beta}}\right\|_{L^{\infty}\left(L^{2}(I)\right)},
$$

it follows that (with the second inequality requiring an integration by parts in $t$ )

$$
\left|\theta_{k}\right|_{1} \leqslant\left\{\begin{array}{l}
C\left\|\frac{\partial^{2} z_{k}}{\partial t^{2}}\right\|_{L^{2}\left(L^{2}(I)\right)}, \quad 2 k \leqslant r-1 \\
C\left[\left\|\frac{\partial^{2} z_{k}}{\partial t^{2}}\right\|_{L^{\infty}\left(H^{-1}(I)\right)}+\left\|\frac{\partial^{3} z_{k}}{\partial t^{3}}\right\|_{L^{1}\left(H^{-1}(I)\right)}\right], \quad 2 k \leqslant r-2 .
\end{array}\right.
$$

Thus,

$$
\left|\theta_{k}\right|_{1} \leqslant \begin{cases}C|w|_{q+2 k+2} h^{q+2 k}, & 2 k \leqslant r-1 \\ \left.\left.C\right|_{w}\right|_{q+r+1} h^{q+r-1}, & r \text { even, } k=(r-2) / 2\end{cases}
$$

THEOREM 8.1. If $\bar{x}=x_{i(n)} \in \Delta_{h}, p_{i(n)}=0$, and $w_{h}$ is determined by (8.2) and (8.6), then for $1 \leqslant q \leqslant r+1$

$$
\left|\left(w-w_{h}\right)(\bar{x}, t)\right| \leqslant \begin{cases}C|w|_{q+2 k+2} h^{q+2 k}, & 2 k \leqslant r-1, \\ C|w|_{q+r+2} h^{q+r-1}, & r \text { even, } k=(r-2) / 2,\end{cases}
$$

and

$$
\left|\left(\frac{\partial w}{\partial t}-\frac{\partial w_{h}}{\partial t}\right)(\bar{x}, t)\right| \leqslant \begin{cases}\left.\left.C\right|_{w}\right|_{q+2 k+3} h^{q+2 k}, & 2 k \leqslant r-1 \\ \left.\left.C\right|_{w}\right|_{q+r+2} h^{q+r-1}, & r \text { even, } k=(r-2) / 2\end{cases}
$$


Proof. The inequality (8.12) follows from the decomposition $w-w_{h}=(w-$ $\left.\tilde{w}_{h}\right)+\theta_{k}-\left(z_{1}+\cdots+z_{k}\right)$, then known [1] superconvergence of the knot value for the two-point boundary problem, (8.11), and (8.8). In order to establish (8.13) it is sufficient to bound $\partial \theta_{k} / \partial t$ in $H^{1}(I)$, since the other terms can be handled as immediately above. First, it follows from $\theta_{k}(0)=0$ and (8.5) that

$$
\left\|\partial^{2} \theta_{k}(0) / \partial t^{2}\right\| \leqslant C\left\|\partial^{2} z_{k}(0) / \partial t^{2}\right\|
$$

If (8.5) is differentiated with respect to time and then $v$ chosen to be $\partial^{2} \theta_{k} / \partial t^{2}$, it is easy to show that

$$
\begin{aligned}
\left\|\frac{\partial^{2} \theta_{k}}{\partial t^{2}}\right\|_{L^{\infty}\left(L^{2}(I)\right)} & +\left\|\frac{\partial \theta_{k}}{\partial t}\right\|_{L^{\infty}\left(H^{1}(I)\right)} \\
& \leqslant \begin{cases}C|w|_{q+2 k+3} h^{q+2 k}, & 2 k \leqslant r-1, \\
C|w|_{q+r+2} h^{q+r-1}, & r \text { even, } k=(r-2) / 2,\end{cases}
\end{aligned}
$$

and (8.13) follows.

Department of Mathematics

The University of Chicago

Chicago, Illinois 60637

Department of Mathematics

The University of Chicago

Chicago, Illinois 60637

Department of Mathematical Sciences

Rice University

Houston, Texas 77001

1. J. DOUGLAS, JR. \& T. DUPONT, "Galerkin approximations for the two point bound ary problem using continuous, piecewise polynomial spaces," Numer. Math., v. 22, 1974, pp. 99109.

2. J. DOUGLAS, JR. \& T. DUPONT, Collocation Methods for Parabolic Equations in a Single Space Variable, Lecture Notes in Math,. vol. 385, Springer-Verlag, Berlin and New York, 1974.

3. J. DOUGLAS, JR., T. DUPONT \& M. F. WHEELER, A Quasi-Projection Approximation Method Applied to Galerkin Procedures for Parabolic and Hyperbolic Equations, Math. Res. Center Rep. \#1465, 1974.

4. J. DOUGLAS, JR., T. DUPONT \& M. F. WHEELER, "A Galerkin procedure for approximating the flux on the boundary for elliptic and parabolic boundary value problems," Rev. Française Automat. Informat. Recherche Operationnelle Sér. Rouge, v. 8, 1974, pp. 47-59.

5. J. DOUGLAS, JR., T. DUPONT \& M. F. WHEELER, Some Superconvergence Results for an $H^{1}$-Galerkin Procedure for the Heat Equation, Lecture Notes in Comput. Sci., vol. 10, Springer-Verlag, Berlin and New York, 1974.

6. T. DUPONT, "Some $L^{2}$ estimates for parabolic Galerkin methods," The Mathematical Foundations of the Finite Element Method with Applications to Partial Differential Equations (A. K. Aziz, Ed.), Academic Press, New York, 1972.

7. H. H. RACHFORD, JR., "Two-level discrete-time Galerkin approximations for second order nonlinear parabolic partial differential equations," SIAM J. Numer. Anal., v. 10, 1973, pp. 1010-1026. 
8. J. A. WHEELER, Simulation of Heat Transfer From a Warm Pipeline Buried in Permafrost, presented to the 74th National Meeting of the American Institute of Chemical Engineers, New Orleans, March, 1973.

9. M. F. WHEELER, "A priori $L_{2}$ error estimates for Galerkin approximations to parabolic partial differential equations," SIAM J. Numer. Anal., v. 10, 1973, pp. 723-759.

10. M. F. WHEELER, " $L_{\infty}$ estimates of optimal order for Galerkin methods for one-dimensional second order parabolic and hyperbolic equations," SIAM J. Numer. Anal., v. 10, 1973, pp. 908-913.

11. M. F. WHEELER, "A Galerkin procedure for estimating the flux for two point boundary problems," SIAM J. Numer. Anal., v. 11, 1974, pp. 764-768.

12. M. F. WHEELER, "An $H^{-1}$ Galerkin method for parabolic equations in a single space variable," SIAM J. Numer. Anal., v. 12, 1975, pp. 803-817. 